\title{
APPLICATIONS AND PROOF OF A UNIQUENESS THEOREM FOR LINEAR INVARIANT FAMILIES OF FINITE ORDER DOUGLAS MICHAEL CAMPBELL
}

Let $S$ be the set of all univalent analytic functions in the open unit disc of the form $f(z)=z+a_{2} z^{2}+\cdots$. One of the most important results concerning such normalized univalent functions, which has not only been extensively used to solve many extremal problems in $S$, but also to demonstrate the essential uniqueness of the solution, is the well known fact that

$$
\left|a_{2}\right| \leqq 2
$$

with equality only for rotations of the Koebe function, $k(z)=$ $z /(1-z)^{2}$.

In this paper we continue our theme [1], [2], [3], [4], that many of the classical results for the family $S$ find not only a more general but also a more meaningful setting in the context of linear invariant families of locally univalent functions of finite order (defined in $\$ 1$ ). The impetus for such an investigation is to be found in Ch. Pommerenke's fundamental papers [13], [14] which form the beginning of this subject.

If $f(z) \in S$, then equality holds in (0.1) only for rotations of the Koebe function, while if $f(z) \in M$, a linear invariant family of finite order $\boldsymbol{\alpha}$, then equality can hold in

$$
\left|a_{2}\right| \leqq \alpha
$$

for functions other than rotations of the generalized Koebe function. Thus we do not always have unique solutions to the extremal problem $\max \left\{\left|a_{2}\right|: f \in M\right\}$. In $\$ 2$, we show that the extremal problem $\max \left\{r\right.$ : each $f$ in $U_{\alpha}$ maps $|z|<r$ univalently onto a convex domain $\}$ does not have a unique solution. Theorem 1 gives an elementary proof that there is a unique solution to the radius of convexity problem in $S$.

Since so many of the uniqueness results for $S$ have classically been derived from the uniqueness properties of inequality (0.1), and in light of the existence of nonunique solutions to extremal problems in $U_{\alpha}$, it is a reasonable question to ask if uniqueness results are possible for the families $U_{\alpha}$. We therefore prove Theorem 2, which is a uniqueness result for the fundamental distortion theorem of $U_{\alpha}$. Theorem 2 is applied in $\S 4$ to show that the generalized Koebe function is the

Received by the editors December 31, 1971 and, in revised form May 9, 1972. AMS 1970 subject classifications. Primary 30A36; Secondary 30A32, 30A26. 
essentially unique solution for many of the extremal problems in the families $U_{\alpha}$. The results of $\S 2$ and 4 suggest that some of the well known uniqueness proofs for $S$ rely on techniques which are 'tricks' peculiar to univalent function theory.

Let $M(r, f)$ denote the maximum modulus of $f(z)$ on $|z|=r$. Hayman [8] and Krzyz [10] investigated the functions $(1-r)^{2} M(r, f)$ and $(1-r)^{3} M\left(r, f^{\prime}\right)$ for $f(z)$ in $S$. In $\$ 5$ we apply the theorems of $\$ 3$ and $\$ 4$ to generalize their results to functions in $U_{\alpha}$. Theorem 8 asserts a piquant relation between $\lim _{r \rightarrow 1}(1-r)^{\alpha} M(r, f)$ and $\lim _{r \rightarrow 1}(1-r)^{\alpha+1} M\left(r, f^{\prime}\right)$ for functions in $U_{\alpha} \cap S$. We conclude with an application of our sharpened distortion theorem to majorizationsubordination theory problems. Theorem 11 and theorem 12 represent a sharpening of our earlier results [4] which are extensions themselves of theorems due to MacGregor [11], Goluzin [5], and Tao Shah [16].

1. Preliminaries. Let $D$ denote the open unit disc. A family of functions $M$ is said to be a linear invariant family if the following two conditions are fulfilled [13, p. 112] :

1. All functions $f(z)$ in $M$ are analytic and locally univalent (that is, $\left.f^{\prime}(z) \neq 0\right)$ in $D$ and have the form $f(z)=z+a_{2} z^{2}+\cdots$.

2. If $\varphi(z)$ is a bilinear map of $D$ onto $D$ and $f(z)$ belongs to $M$, then the function

$$
\Lambda_{\varphi}[f(z)]=\frac{f(\varphi(z))-f(\varphi(0))}{\varphi^{\prime}(0) f^{\prime}(\varphi(0))}=z+\cdots
$$

must also belong to $M$.

The order of a linear invariant family $M$ is defined as $\alpha=$ $\sup \left\{\left|f^{\prime \prime}(0) / 2\right|: f \in M\right\}$, or equivalently [13, p. 115],

$$
\alpha=\sup _{f \in M} \sup _{z \in D}\left\{\left|-\bar{z}+(1 / 2)\left(1-|z|^{2}\right) f^{\prime \prime}(z) / f^{\prime}(z)\right|\right\} .
$$

The order of a linear invariant family is never less than 1 [13, p. 117].

Let $U_{\alpha}(1 \leqq \alpha<\infty)$ be the universal linear invariant family of order $\alpha$; that is, the union of all linear invariant families of order $\leqq \alpha$. The family $U_{1}$ is precisely the family of all normalized convex univalent functions [13, p. 134]. Close-to-convex functions of order $\beta$ are contained in $U_{\beta+1}$ [15]. Functions whose boundary rotation is bounded by $A$ are contained in $U_{\alpha}$ where $\alpha=A / 2 \pi$ [1, p. 57]. The family $S$, of all normalized univalent functions, is in $U_{2}[13$, p. 115]. Each family $U_{\alpha}(\alpha>1)$ contains functions of infinite valence; for example, the function

$$
f(z)=\frac{1}{2 i \gamma}\left[\left(\frac{1+z}{1-z}\right)^{i \gamma}-1\right], \gamma>0,
$$


has infinite valence and has order $\left(1+\gamma^{2}\right)^{1 / 2}[13$, p. 128]

The Koebe function, $k(z)=z /(1-z)^{2}$, is the unique solution to many extremal problems in $S$. The natural generalization of the Koebe function to $U_{\alpha}$ is

$$
f(z)=\frac{1}{2 \alpha}\left[\left(\frac{1+z}{1-z}\right)^{\alpha}-1\right],\left(f \in U_{\alpha}\right) .
$$

For the special case $\alpha=2$ in (1.2), we recover the Koebe function. If $\alpha=1$, we obtain $z /(1-z)$, the function which is the essentially unique extremal in many questions concerning convex univalent functions.

We say that $g(z)$ is a rotation of $f(z)$, if $g(z)=e^{-i \theta} f\left(e^{i \theta} z\right)$, $\theta \in[0,2 \pi]$.

The ultra-classical distortion theorem for $S$ asserts that for each $f(z)$ in $S$

$$
(1-|z|) /(1+|z|)^{3} \leqq\left|f^{\prime}(z)\right| \leqq(1+|z|) /(1-|z|)^{3}
$$

and equality holds in (1.3) for any nonzero $z$ in $D$ if and only if $f(z)$ is a rotation of the Koebe function. The proofs concerning equality in (1.3) generally are based on the fact that for $f(z)$ in $S,\left|a_{2}\right|$ is equal to 2 if and only if $f(z)$ is a rotation of the Koebe function [12, p. 19], $[5$, p. 51$],[9$, p. 4]. From this it also follows that in the class $S$, the unique solutions for the extremal problems

(1.4) $\max _{|z|=r}|f(z)|, \min _{|z|=r}|f(z)|, \max _{|z|=r}|f(z)| f^{\prime}(z)\left|, \min _{|z|=r}\right| f(z)\left|f^{\prime}(z)\right|$,

$$
\max \{r:|w|<r \text { is contained in } f(D), f \in S\}
$$

are the rotations of the Koebe function.

2. Extremal Problems with Non-Unique Solutions. It is true that the function (1.2) and its rotations are extremal functions for the problems $\max \left|f^{\prime \prime}(0) / 2\right|, \max |f(z)|, \max \left|f^{\prime}(z)\right|, \min \left|f^{\prime}(z)\right|$ in the class $U_{\alpha}$. In addition they are extremal functions for $\min |f(z)|$, $\max \left|f^{\prime}(z)\right| f(z)|, \min | f^{\prime}(z) / f(z) \mid$ for functions $f(z)$ in $S \cap U_{\alpha}$.

However, there are functions which are in $U_{\alpha}$ other than rotations of the generalized Koebe function for which $\left|a_{2}\right|$ is equal to $\alpha$. For example, the function

$$
g(z)=\left(e^{a z}-1\right) / a, \quad a=\alpha+\left(\alpha^{2}-1\right)^{1 / 2}, \quad \alpha>1,
$$

can be easily shown to be in $U_{\alpha}[3$, p. 709]. Yet if we let $\varphi(z)=(z-1 / a) /(1-z / a)$, a bilinear map of $D$ onto $D$, and compute the second coefficient of $h(z)=\Lambda_{\varphi}[g(z)]$, then we obtain 
$\left|h^{\prime \prime}(0) / 2\right|=\alpha$. The functions $\Lambda_{\varphi}[g(z)]$ and (1.2) are by no means equivalent.

The nonuniqueness of extremals for the problem $\sup \left\{\left|a_{2}\right|: f \in U_{\alpha}\right\}$, $\alpha>1$, has interesting consequences for such simple problems as the radius of convexity of a linear invariant family. Pommerenke has shown, under the superfluous assumption that the family is compact, that the radius of convexity of a linear invariant family $M$ of order $\alpha$ is precisely $\alpha-\left(\alpha^{2}-1\right)^{1 / 2}$ [13, p. 133]. Consequently, the radius of convexity of $U_{2}$ is $2-\sqrt{3}$, the same as the radius of convexity of $S$ which is a proper subset of $U_{2}$.

Although Gronwall claimed that only rotations of the Koebe function take on this minimum radius of convexity in $S$ [6, p. 251], there is apparently no proof of this fact in the literature. Therefore the following elementary proof is included.

THEOREM 1. If $f(z) \in S$ has radius of convexity $2-\sqrt{3}$, then $f(z)$ is a rotation of the Koebe function.

Proof. Since the radius of convexity is $2-\sqrt{3}=r$, there is a point on $|z|=r$ for which

$$
\operatorname{Re}\left(1+z f^{\prime \prime}(z) / f^{\prime}(z)\right)=0 .
$$

By a rotation of $f(z)$, if necessary, we may assume that (2.2) holds for the point $z=-r$. If we calculate the second coefficient of $\Lambda_{\varphi}[f(z)]$, where $\varphi(z)=(z-r) /(1-r z)$, then we obtain

$$
\begin{aligned}
a_{2} & =r+(1 / 2)\left(1-r^{2}\right) f^{\prime \prime}(-r) / f^{\prime}(-r) \\
& =\frac{1+r^{2}}{2 r}-\frac{1-r^{2}}{2 r}\left(1-r \frac{f^{\prime \prime}(-r)}{f^{\prime}(-r)}\right) .
\end{aligned}
$$

A computation shows that $\left(1+r^{2}\right) / 2 r=2$. Thus with (2.2) and the fact that $\Lambda_{\varphi}[f(z)]$ is again in $S$, we can conclude that

$$
2 \geqq\left|a_{2}\right|=\left|2-\left(\frac{1-r^{2}}{2 r}\right) i \operatorname{Im}\left(1-r \frac{f^{\prime \prime}(-r)}{f^{\prime}(-r)}\right)\right| \geqq 2 .
$$

Therefore, $a_{2} \equiv 2$ and hence $\Lambda_{\varphi}[f(z)]$ is the Koebe function. The Koebe function has the peculiar property that $\Lambda_{\varphi}[k(z)]=k(z)$ for all $\varphi(z)=(z+r) /(1+r z), \quad-1<r<1$. Hence, $f(z)=\Lambda_{I d}[f(z)]=$ $\Lambda_{\varphi \circ \varphi^{-1}}[f(z)]=\Lambda_{\varphi-1}\left[\Lambda_{\varphi}[f(z)]\right]=\Lambda_{\varphi-1}[k(z)]=k(z)$, (where we have used the facts (1) $\mathbb{Q}$, the set of all Möbius transformations of $D$ onto $D$, is a group and (2) $\Lambda_{\varphi}\left[\Lambda_{\psi}[f]\right]=\Lambda_{\psi \circ \varphi}[f]$ for all $\Psi, \varphi$ in $\mathfrak{l}$.) This concludes the proof of the theorem. 
The phenomenon of unique (up to rotation) extremal functions is completely lost for every $U_{\alpha}$ class for the radius of convexity problem. The function $g(z)$ in $(2.1)$ is in $U_{\alpha}$ and has radius of convexity $\alpha-\left(\alpha^{2}-1\right)^{1 / 2}$. The function $f(z)$ in (1.2) is also in $U_{\alpha}$ and has this same minimum possible radius of convexity. Yet the functions $f(z)$ and $g(z)$ are fundamentally different; the function $f(z)$ is rational and has finite valence in $|z|<\infty$; the function $g(z)$ is entire and has infinite valence in $|z|<\infty$. (Consequently, we can never even have $f(z)=$ $\left.\Lambda_{\varphi}[g(z)], \varphi(z) \in \mathfrak{R}\right)$.

3. A Sharpened Distortion Theorem for $U_{\alpha}$. Since it is the uniqueness of extremals in the solution to the problem $\sup \left\{\left|a_{2}\right|: f \in S\right\}$ that has been used to prove the uniqueness of solutions in Theorem 1 and the extremal problems of (1.4) and (1.5), the following theorems are by no means obvious in light of the preceding observations. We include the first part of Theorem 2, which is due to Pommerenke [13, p. 116], for completeness.

Theorem 2. Let $f(z) \in U_{\alpha}$. Then

$$
\frac{(1-|z|)^{\alpha-1}}{(1+|z|)^{\alpha+1}} \leqq\left|f^{\prime}(z)\right| \leqq \frac{(1+|z|)^{\alpha-1}}{(1-|z|)^{\alpha+1}} .
$$

Equality holds in (3.1) for any point $0<|z|<1$ if and only if $f(z)$ is a rotation of $(1.2)$.

Proof. The function $f(z)$ is in $U_{\alpha}$ if and only if

$$
\left|-\bar{z}+(1 / 2)\left(1-|z|^{2}\right) f^{\prime \prime}(z) / f^{\prime}(z)\right| \leqq \alpha, z \in D,
$$

which is equivalent to

$$
\left|\frac{\partial}{\partial r} \log \left(1-|z|^{2}\right) f^{\prime}(z)\right| \leqq \frac{2 \alpha}{1-r^{2}}, \quad z=r e^{i \theta} .
$$

An integration yields $\left|\log \left(1-|z|^{2}\right) f^{\prime}(z)\right| \leqq \alpha \log [(1+r) /(1-r)]$ which implies (3.1).

Clearly, if $f(z)$ is (1.2), then equality holds on the right hand side of (3.1) for $z=r$ and on the left hand side for $z=-r$.

On the other hand, suppose that equality holds on the right hand side of (3.1) for some $z=r e^{i \theta}, 0<|z|<1$. By considering $e^{i \theta} f\left(e^{-i \theta} z\right)$, we may assume $z=r>0$ and therefore that $\left|f^{\prime}(r)\right|=(1+r)^{\alpha-1 /}$ $(1-r)^{\alpha+1}$. If

$$
\begin{aligned}
u(x)= & \log \left|f^{\prime}(x)\right|-(\alpha-1) \log (1+x) \\
& +(\alpha+1) \log (1-x), \quad 0 \leqq x<1,
\end{aligned}
$$


then we claim that $u^{\prime}(x)=\operatorname{Re}\left(f^{\prime \prime}(x) / f^{\prime}(x)\right)-(2 \alpha+2 x) /\left(1-x^{2}\right)$ is nonpositive for $0<x<1$. For if $u^{\prime}\left(x_{0}\right)$ were positive for some $x_{0}$ in $(0,1)$, then

$$
\begin{aligned}
\alpha & =\sup _{z \in D}\left|-\bar{z}+(1 / 2)\left(1-|z|^{2}\right) f^{\prime \prime}(z)\right| f^{\prime}(z) \mid \\
& \geqq\left|-x_{0}+(1 / 2)\left(1-x_{0}{ }^{2}\right) f^{\prime \prime}\left(x_{0}\right) / f^{\prime}\left(x_{0}\right)\right| \\
& =\left|\alpha+(1 / 2)\left(1-x_{0}{ }^{2}\right) u^{\prime}\left(x_{0}\right)\right|>\alpha,
\end{aligned}
$$

which is absurd. Since $u(0)=u(r)=0$, and $u^{\prime}(x) \leqq 0$, we must consequently have $u(x) \equiv 0$ on $[0, r]$. Let

$$
\begin{aligned}
F(z) & =\log f^{\prime}(z)-(\alpha-1) \log (1+z)+(\alpha+1) \log (1-z) \\
& =u(z)+i v(z) .
\end{aligned}
$$

We have for $x$ in $[0, r]$ the equalities

$$
\begin{aligned}
\left|-x+\left(\frac{1}{2}\right)\left(1-x^{2}\right) f^{\prime \prime}(x) / f^{\prime}(x)\right| \\
=\left|-x+\frac{1}{2}\left(1-x^{2}\right)\left(F^{\prime}(x)-\frac{(2 \alpha+2 x)}{1-x^{2}}\right)\right| \\
=\left|\alpha-(1 / 2)\left(1-x^{2}\right) i v^{\prime}(x)\right|,
\end{aligned}
$$

because $u^{\prime}(x) \equiv 0$ on $[0, r]$. Upon remembering that $f(z) \in U_{\alpha}$, we can therefore conclude just as above that $v^{\prime}(x) \equiv 0$ on $[0, r]$. Since $F^{\prime}(z)$ is analytic and identically zero on $[0, r]$, we have

$$
F^{\prime}(z)=f^{\prime \prime}(z) / f^{\prime}(z)-(2 \alpha+2 z) /\left(1-z^{2}\right) \equiv 0, z \in D .
$$

The solution to the differential equation (3.3) is the function (1.2). A similar argument holds for equality on the left hand side of (3.1).

4. Applications to $U_{\alpha}$ Theory. If we apply Theorem 2 to Pommerenke's proofs of Theorem 1.1 and Lemma 1.3 of [13], then, in his notation, we have:

THEOREM 3. Let $M$ be a linear invariant family of order $\alpha$. If $f(z) \in M$ and $|z|=r<1$, then

$$
\frac{1}{2 \alpha}\left[1-\left(\frac{1-r}{1+r}\right)^{\alpha}\right] \leqq \min \left[d(f(z), 0), \frac{d(f(z), 0)}{\left(1-|z|^{2}\right)\left|f^{\prime}(z)\right|}\right]
$$

$$
\max \left[\frac{\ell(f(z), 0)}{\left(1-|z|^{2}\right)\left|f^{\prime}(z)\right|}, \ell(f(z), 0)\right] \leqq \frac{1}{2 \alpha}\left[\left(\frac{1+r}{1-r}\right)^{\alpha}-1\right]
$$




$$
(2 \alpha)^{-1}\left(1-|z|^{2}\right)\left|f^{\prime}(z)\right| \leqq d(f(z)) .
$$

Furthermore, the radius $R$ of the largest schlicht disc centered at $f(0)=0$ and contained in $\{f(z):|z| \leqq r\}$ satisfies

$$
R \geqq \frac{1}{2 \alpha}\left[1-\left(\frac{1-r}{1+r}\right)^{\alpha}\right] .
$$

In particular, the radius of the largest schlicht disc centered at 0 and contained in the Riemann Surface $f(D)$ is $\geqq(2 \alpha)^{-1}$. Equality holds in any of the above inequalities (if $z$ is a nonzero element of $D$ ) if and only if $f(z)$ is a rotation of $(1.2)$.

For the special case of $\alpha=2$, we obtain a generalization of the classic $1 / 4$ covering theorem for $S$, since $S$ is a proper subset of $U_{\alpha}$. The case $\alpha=1$ yields the $1 / 2$ covering theorem for the convex univalent functions.

One cannot give a lower bound for $|f(z)|$ for an arbitrary function $f(z)$ in $U_{\alpha}$ and all $z$ in $D$, since $f(z)$ can have zeros for $z \neq 0$. However, we can prove the following two useful results. We first remark that the radius of univalence, $R_{u}$, of a family $M$ is the supremum of the set of $r$ in $[0,1]$ such that each $f$ in $M$ is univalent in $|z|<r$. If $M$ is a linear invariant family of order $\alpha$, then $R_{u}(M)$ is no less than $1 / \alpha$ [13, p. 134].

THEOREM 4. Let $M$ be a linear invariant family of order $\alpha, 1 \leqq \alpha$ $<\infty$, with radius of univalance $R_{u}$. If $f(z) \in M$, then for all $|z|<R_{u}$,

(4.5) $\frac{1}{2 \alpha}\left[1-\left(\frac{1-|z|}{1+|z|}\right)^{\alpha}\right] \leqq \min \left[|f(z)|, \frac{|f(z)|}{\left(1-|z|^{2}\right)\left|f^{\prime}(z)\right|}\right]$. If $f(z) \in S \cap U_{\alpha}$, we have for all $z$ in $D$

$$
\begin{aligned}
\frac{1}{2 \alpha}\left[1-\left(\frac{1-|z|}{1+|z|}\right)^{\alpha}\right] & \leqq \frac{|f(z)|}{\left(1-|z|^{2}\right)\left|f^{\prime}(z)\right|} \\
& \leqq \frac{1}{2 \alpha}\left[\left(\frac{1+|z|}{1-|z|}\right)^{\alpha}-1\right] .
\end{aligned}
$$

Equality holds in (4.5) and (4.6) for nonzero $z$ in $D$ if and only if $f(z)$ is a rotation of $(1.2)$.

Proof. From (4.1) we know that for $|z|=r$,

$$
\frac{1}{2 \alpha}\left[1-\left(\frac{1-r}{1+r}\right)^{\alpha}\right] \leqq d(f(z), 0) .
$$


Since $d(f(z), 0) \geqq|f(z)|$ is true for all $z$ in $D$, if $|z|$ is within the radius of univalence for the family, then it is easy to check that

$$
\min _{|z|=r}|f(z)|=\min _{|z|=r} d(f(z), 0), \quad|z|<R_{u} .
$$

Therefore, for all $f(z)$ in $M$ and $|z|<R_{u}$,

$$
\frac{1}{2 \alpha}\left[1-\left(\frac{1-|z|}{1+|z|}\right)^{\alpha}\right] \leqq|f(z)| .
$$

The function $\Lambda_{\varphi}[f(z)]$, where $\varphi(z)=(z+\zeta) /(1+\bar{\zeta} z)$ and $|\zeta|<R_{u}$, is in the family $M$ because $M$ is a linear invariant family. If we apply inequality (4.7) to the function $\Lambda_{\varphi}[f(z)]$ at the point $z=-\zeta$, we obtain the other part of inequality (4.5). The inequality (4.6), which yields as special cases the well known inequalities for the convex functions and the univalent functions, follows immediately from (4.5) and (4.2). Upon noting that $\ell(f(z), 0) \geqq|f(z)|$, the last claim of the theorem follows quickly from Theorem 3 .

5. Applications to Maximum Modulus Problems. Let $M(r, f)$ denote the maximum modulus of $f(z)$ on $|z|=r$. In 1951 Hayman showed that for $f(z)$ in $S, M(r, f)(1-r)^{2} / r$ is a strictly decreasing function of $r$ unless $f(z)$ is a rotation of the Koebe function [8]. In particular $\lim _{r \rightarrow 1}(1-r)^{2} M(r, f)$ exists and is $\leqq 1$. Hayman later proved [9, p. 100] that if $f(z)$ is a circumferentially mean $p$-valent function in $D$, then $\lim _{r \rightarrow 1}(1-r)^{2 p} M(r, f)$ exists finitely. Krzyz in 1955 extended Hayman's results in a different direction. If $f(z) \in S$, then $M\left(r, f^{\prime}\right)(1-r)^{3} /(1+r)$ is a strictly decreasing function of $r$ unless $f(z)$ is a rotation of the Koebe function. He also showed that for $f$ in $S$

$$
\lim _{r \rightarrow 1}(1-r)^{2} M(r, f)=2 \lim _{r \rightarrow 1}(1-r)^{3} M\left(r, f^{\prime}\right) .
$$

We extend their developments into general $U_{\alpha}$ theory. Lemma 5 is due to Krzyz [10].

Lemma 5. Let $f(z)$ be analytic in $D$. Let $\Psi(r)$ be a positive differentiable function of $r$ in $(0,1)$. If

$$
\left|f^{\prime}(z) / f(z)\right| \leqq \Psi^{\prime}(|z|) / \Psi(|z|), \quad z \neq 0, \quad z \in D,
$$

then

$$
\begin{gathered}
\Psi(r)^{-1}\left|f\left(r e^{i \theta}\right)\right| \quad(\theta \text { fixed }) \\
\Psi(r)^{-1} M(r, f)
\end{gathered}
$$


are both nonincreasing functions of $r$. Furthermore, the functions in (5.2) and (5.3) are strictly decreasing if strict inequality holds in (5.1).

Theorem 6. If $f(z) \in U_{\alpha}, 1 \leqq \alpha<\infty$, then

$$
\begin{gathered}
(1+r)^{1-\alpha}(1-r)^{\alpha+1}\left|f^{\prime}\left(r e^{i \theta}\right)\right|, \quad(\theta \text { fixed }), \\
(1+r)^{1-\alpha}(1-r)^{\alpha+1} M\left(r, f^{\prime}\right),
\end{gathered}
$$

are both nonincreasing functions of $r$ which tend to limits $A$ and $B$ respectively $(0 \leqq A \leqq B \leqq 1)$ as $r$ approaches 1 . Moreover, $A=1$ if and only if $f(z)=e^{i \theta} g\left(e^{-i \theta} z\right)$ where $g(z)$ is the function (1.2). Similarly, $B=1$ if and only if $f(z)$ is a rotation of the function (1.2). Finally, the functions in (5.4) and (5.5) are both strictly decreasing unless $f(z)$ is a rotation of $(1.2)$.

Proof. For $r$ in $(0,1), \Psi(r)=(1+r)^{\alpha-1}(1-r)^{-(1+\alpha)}$ is a positive differentiable function of $r$. Furthermore, for each $f(z)$ in $U_{\alpha}$, it follows from (1.1) that

$$
\left|f^{\prime \prime}(z) / f^{\prime}(z)\right| \leqq 2(\alpha+|z|) /\left(1-|z|^{2}\right) \equiv \Psi^{\prime}(|z|) / \Psi(|z|) .
$$

The conclusion of Lemma 5 implies that the functions in (5.4) and (5.5) are nonincreasing. The inequalities $0 \leqq A \leqq B \leqq 1$ follow immediately from $B \leqq 1$. To show $B \leqq 1$ it suffices to demonstrate that

$$
\lim _{r \rightarrow 0^{+}}(1+r)^{1-\alpha}(1-r)^{\alpha+1} M\left(r, f^{\prime}\right)=1 .
$$

This is an easy consequence of the fact that for $r$ sufficiently small, $M\left(r, f^{\prime}\right)=1+2\left|a_{2}\right| r+\cdots[7$, p. 142]. Finally, suppose that $B=1$. Then for each $r$ in $[0,1]$

$$
\begin{aligned}
1 & =(1-r)^{\alpha+1}(1+r)^{1-\alpha} M\left(r, f^{\prime}\right) \\
& =(1-r)^{\alpha+1}(1+r)^{1-\alpha}\left|f^{\prime}\left(r e^{i \theta}\right)\right| \quad(\theta=\theta(r)) .
\end{aligned}
$$

Consequently, by Theorem $2, f(z)$ must be a rotation of (1.2). The proof for the case $A=1$ follows immediately.

In an entirely analogous manner, with $\Psi(r)=[(1+r) /(1-r)]^{\alpha}-1$, we can establish the following result.

THEOREM 7. If $f(z) \in S \cap U_{\alpha}, 1 \leqq \alpha \leqq 2$, then

$$
\begin{aligned}
& \left\{\frac{1}{2 \alpha}\left[\left(\frac{1+r}{1-r}\right)^{\alpha}-1\right]\right\}^{-1}\left|f\left(r e^{i \theta}\right)\right| \quad(\theta \text { fixed }) \\
& \left\{\frac{1}{2 \alpha}\left[\left(\frac{1+r}{1-r}\right)^{\alpha}-1\right]\right\}^{-1} M(r, f)
\end{aligned}
$$


are both nonincreasing functions which tend to $A$ and $B$ respectively $(0 \leqq A \leqq B \leqq 1)$ as $r \rightarrow 1$. Moreover $A=1$ if and only if $f(z)=$ $e^{i \theta} g\left(e^{-i \theta} z\right)$ where $g(z)$ is the function (1.2). Similarly, $B=1$ if and only if $f(z)$ is a rotation of (1.2). Finally, the functions in (5.6) and (5.7) are strictly decreasing unless $f(z)$ is a rotation of (1.2).

If $f(z) \in S \cap U_{\alpha}$, then not only do the functions in (5.6) and (5.7) approach limits as $r$ tends to 1 , but these limits satisfy an extremely elementary functional relationship which is dependent only on $\alpha$, and not on the function $f(z)$. This observation generalizes the result of Krzyz and explains the significance of the constants in Krzyz's theorem.

TheOREM 8. If $f(z) \in S \cap U_{\alpha}, 1 \leqq \alpha \leqq 2$, then the following limits exist:

$$
\lim _{r \rightarrow 1}(1-r)^{\alpha}\left|f\left(r e^{i \theta}\right)\right|=A_{f, \theta}, \quad \lim _{r \rightarrow 1}(1-r)^{\alpha} M(r, f)=B_{f},
$$

(5.9) $\lim _{r \rightarrow 1}(1-r)^{\alpha+1}\left|f^{\prime}\left(r e^{i \theta}\right)\right|=A_{f, \theta}^{*}, \lim _{r \rightarrow 1}(1-r)^{\alpha+1} M\left(r, f^{\prime}\right)=B_{f}^{*}$,

where $\theta \in[0,2 \pi]$ is fixed but arbitrary. We necessarily have

$$
\begin{gathered}
\alpha A_{f, \theta}=A_{f, \theta}^{*}, \\
\alpha B_{f}=B_{f}^{*}, \\
0 \leqq A_{f, \theta} \leqq B_{f} \leqq 2^{\alpha-1} / \alpha, \\
0 \leqq A_{f, \theta}^{*} \leqq B_{f}^{*} \leqq 2^{\alpha-1} .
\end{gathered}
$$

Furthermore, $A_{f, \theta}=2^{\alpha-1} / \alpha, \quad A_{f, \theta}^{*}=2^{\alpha-1}$ if and only if $f(z)=$ $e^{i \theta} g\left(e^{-i \theta} z\right)$ where $g(z)$ is (1.2). Finally, $B_{f}=2^{\alpha-1 / \alpha}, B_{f}^{*}=2^{\alpha-1}$ if and only if $f(z)$ is a rotation of $(1.2)$.

Proof. We shall indicate the proof for $B_{f}$ and $B_{f}{ }^{*}$ only. The proof of the results concerning $A_{f, \theta}$ and $A_{f, \theta}^{*}$ is identical. Theorem 6 and Theorem 7 imply the existence of the limits (5.8) and (5.9), the inequalities (5.12) and (5.13), and the fact that $B_{f}=2^{\alpha-1} / \alpha, B_{f}^{*}=2^{\alpha-1}$ if and only if $f(z)$ is a rotation of (1.2).

We now prove that $B_{f}$ and $B_{f}^{*}$ are always related by $\alpha B_{f}=B_{f}{ }^{*}$. We begin by demonstrating that for any function $\mathrm{f}(z)$ analytic in $D$ and any $\gamma>0$,

$$
\limsup _{r \rightarrow 1}(1-r)^{\gamma} M(r, f)=b_{f}
$$


implies

$$
b_{f}^{*}=\limsup _{r \rightarrow 1}(1-r)^{\gamma+1} M\left(r, f^{\prime}\right) \geqq \gamma b_{f} .
$$

Assume on the contrary that $b_{f}{ }^{*}<\gamma b_{f}$. Choose $\epsilon>0$ such that $\left(b_{f}{ }^{*}+\epsilon\right) \gamma^{-1}<b_{f}$. From the definition of $b_{f}{ }^{*}$, there is an $r(\epsilon)$ such that for all $|z|>r(\epsilon),\left|f^{\prime}(z)\right| \leqq\left(b_{f}^{*}+\epsilon\right)(1-r)^{-\gamma-1}$. Integrating along a radius, we obtain for any $r>r(\epsilon)$ the inequality

$$
M(r, f) \leqq M(r(\epsilon), f)+\left(b_{f}^{*}+\epsilon\right) \gamma^{-1}(1-r)^{-\gamma},
$$

which implies that

$$
b_{f}=\limsup _{r \rightarrow 1}(1-r)^{\gamma} M(r, f) \leqq\left(b_{f}^{*}+\epsilon\right) \gamma^{-1}<b_{f},
$$

which is absurd. In particular, for $\gamma=\alpha$ and $f(z)$ in $S \cap U_{\alpha}$, since the limits exist in (5.14) and (5.15), we obtain $B_{f}{ }^{*} \geqq \alpha B_{f}$. On the other hand, from inequality (4.6), we have for all $z$ in $D$

$$
\frac{1}{2 \alpha}\left[1-\left(\frac{1-r}{1+r}\right)^{\alpha}\right]\left|f^{\prime}(z)\right| \leqq\left(1-r^{2}\right)^{-1}|f(z)|,
$$

which, upon taking $M(r, f)$ and then $M\left(r, f^{\prime}\right)$, implies that

$$
\frac{1}{2 \alpha}\left[1-\left(\frac{1-r}{1+r}\right)^{\alpha}\right] M\left(r, f^{\prime}\right) \leqq\left(1-r^{2}\right)^{-1} M(r, f) .
$$

Inequality (5.16) is equivalent to

$$
2 \alpha(1-r)^{\alpha} M(r, f)-(1-r)^{\alpha+1} M\left(r, f^{\prime}\right)(1+r)\left[1-\left(\frac{1-r}{1+r}\right)^{\alpha}\right] \geqq 0 .
$$

Therefore, upon taking the limit as $r \rightarrow 1$, we have $\alpha B_{f} \geqq B_{f}^{*}$. Thus, $\alpha B_{f}=B_{f}^{*}$ which concludes the theorem.

Corollary 9. If $f(z) \in S$, and $\theta$ is arbitrary but fixed, then

$$
\begin{aligned}
& 2 \lim _{r \rightarrow 1}(1-r)^{2} M(r, f)=\lim _{r \rightarrow 1}(1-r)^{3} M\left(r, f^{\prime}\right) \leqq 1, \\
& 2 \lim _{r \rightarrow 1}(1-r)^{2}\left|f\left(r e^{i \theta}\right)\right|=\lim _{r \rightarrow 1}(1-r)^{3}\left|f^{\prime}\left(r e^{i \theta}\right)\right| \leqq 1 .
\end{aligned}
$$

Corollary 10. If $f(z)$ is a convex univalent function, and $\theta$ is arbitrary but fixed, then

$$
\begin{aligned}
\lim _{r \rightarrow 1}(1-r) M(r, f) & =\lim _{r \rightarrow 1}(1-r)^{2} M\left(r, f^{\prime}\right) \leqq 1, \\
\lim _{r \rightarrow 1}(1-r)\left|f\left(r e^{i \theta}\right)\right| & =\lim _{r \rightarrow 1}(1-r)^{2}\left|f^{\prime}\left(r e^{i \theta}\right)\right| \leqq 1 .
\end{aligned}
$$

The results of Corollary 9 were first proved by Krzyz [10]. The results of Corollary 10 are apparently new for the convex univalent 
functions. We can apply Theorem 8 to close-to-convex functions of order $\boldsymbol{\beta}$ and also to univalent functions of bounded boundary rotation to obtain new results for these families.

We have not been able to extend Theorem 7 to arbitrary $U_{\alpha}$ classes. Such an extension would allow us to generalize the piquant relation (5.10) and (5.11) of Theorem 8. We conjecture that Theorem 7 and Theorem 8 can be extended to functions of bounded boundary rotation. Since the family of all functions of bounded boundary rotation is dense in $X=\cup\left\{U_{\alpha}: \alpha \geqq 1\right\}$, this would be a marked extension. The generalized Koebe function is of bounded boundary rotation. To give a basis for the conjecture and to provide examples of functions in $U_{\alpha}$ such that $A_{f, \theta}\left(A_{f, \theta}^{*}\right)$ takes on an arbitrary value in $\left[0,2^{\alpha-1} / \alpha\right]$ $\left(\left[0,2^{\alpha-1}\right]\right)$, we further examine the function (1.2).

If $\varphi(z)=(z+\zeta) /(1+\bar{\zeta} z),|\zeta|<1$ and $f(z)$ is (1.2), then a routine computation yields

$$
\Lambda_{\varphi}[f(z)]=\frac{1}{2 \alpha} \cdot \frac{1-\zeta^{2}}{1-|\zeta|^{2}}\left[\left(\frac{1+z\left(\frac{1+\bar{\zeta}}{1+\zeta}\right)}{1-z\left(\frac{1-\bar{\zeta}}{1-\zeta}\right)}\right)^{\alpha}-1\right] .
$$

Let $(1+\bar{\zeta}) /(1+\zeta)=e^{i \beta},(1-\bar{\zeta}) /(1-\zeta)=e^{i \gamma}$. It is easy to check that $\left(e^{i \beta}+e^{i \gamma}\right) / 2=\left(1-|\zeta|^{2}\right) /\left(1-\zeta^{2}\right)$. Hence,

$$
\begin{aligned}
g(z)=\Lambda_{\varphi}[f(z)] & =\frac{1}{\alpha} \cdot \frac{1}{\left(e^{i \gamma}+e^{i \beta}\right)}\left[\left(\frac{1+z e^{i \beta}}{1-z e^{i \gamma}}\right)^{\alpha}-1\right], \\
g^{\prime}(z) & =\left(1+z e^{i \beta}\right)^{\alpha-1} /\left(1-z e^{i \gamma}\right)^{\alpha+1} .
\end{aligned}
$$

It follows immediately that

$$
\begin{aligned}
& A=\lim _{r \rightarrow 1}(1-r)^{\alpha}\left|g\left(r e^{-i \gamma}\right)\right|=\left|1+e^{i(\beta-\gamma)}\right|^{\alpha-1} / \alpha, \\
& B=\lim _{r \rightarrow 1}(1-r)^{\alpha+1}\left|g^{\prime}\left(r e^{-i \gamma}\right)\right|=\left|1+e^{i(\beta-\gamma)}\right|^{\alpha-1},
\end{aligned}
$$

and $\alpha A=B$. Although $\beta$ and $\gamma$ are not independent, we do have

$$
\begin{aligned}
e^{i(\beta-\gamma)} & =\left(\frac{1-\zeta}{1+\zeta}\right)\left(\frac{1+\bar{\zeta}}{1-\bar{\zeta}}\right), \\
& =r e^{i \theta}\left(e^{i \theta} / r\right)=e^{2 i \theta} .
\end{aligned}
$$

Since we can choose $\zeta$ so that $\theta \in(-\pi / 2, \pi / 2)$, we see that $\beta-\gamma$ can be made to be any value in $(-\pi, \pi)$ and our claim follows.

6. Applications to majorization-subordination theory. Let $f(z), F(z)$ and $\varphi(z)$ be functions analytic in $|z|<r$. We say that $f(z)$ is majorized 
by $F(z)$ in $|z|<r$, if $|f(z)| \leqq|F(z)|$ in $|z|<r$. We say that $f(z)$ is subordinate to $F(z)$ in $|z|<r$, if $f(z)=F(\varphi(z))$ where $|\varphi(z)| \leqq|z|$ in $|z|<r$. In a recent paper [4], we generalized several of the majorization-subordination theorems of univalent function theory to $U_{\alpha}$. We showed that two of the theorems yielded 'best possible' results. Since these particular theorems are based on the inequalities (3.1) or (4.6), we can now make our earlier theorems even more precise.

THEOREM 11. let $f(z)$ be majorized by $F(z)$ in $D$. If $F(z) \in U_{\alpha}$, $1 \leqq \alpha<\infty$, then $f^{\prime}(z)$ is majorized by $F^{\prime}(z)$ in

$$
|z| \leqq \frac{(\alpha+1)^{1 / \alpha}-1}{(\alpha+1)^{1 / \alpha}+1}=\tanh \left[(2 \alpha)^{-1} \ln (\alpha+1)\right] .
$$

The result is best possible for each $\alpha$. Moreover, $\left|f^{\prime}(z)\right|<\left|F^{\prime}(z)\right|$ in $|z|<\tanh \left[(2 \alpha)^{-1} \ln (\alpha+1)\right]$ unless $f(z)=e^{i \theta} F(z)$. Suppose that $F(z) \in U_{\alpha}$ is not a rotation of (1.2). If $f(z)$ is majorized by $F(z)$ in $D$, then $f^{\prime}(z)$ is majorized by $F^{\prime}(z)$ in $|z|<R$ for some $R>$ $\tanh \left[(2 \alpha)^{-1} \ln (\alpha+1)\right]$, where $R$ depends only on $F(z)$ and not on $f(z)$.

TheOREM 12. Let $f(z)$ be subordinate to $F(z)$ in $D$. If $f^{\prime}(0) \geqq 0$ and $F(z) \in U_{\alpha}, 1.65 \leqq \alpha<\infty$, then $f^{\prime}(z)$ is majorized by $F^{\prime}(z)$ in

$$
|z| \leqq(\alpha+1)-\left(\alpha^{2}+2 \alpha\right)^{1 / 2}
$$

The result is best possible for each $\alpha$. Moreover, $\left|f^{\prime}(z)\right|<\left|F^{\prime}(z)\right|$ in $|z|<(\alpha+1)-\left(\alpha^{2}+2 \alpha\right)^{1 / 2}$ unless $f(z) \equiv F(z)$. Suppose that $f(z) \in U_{\alpha}$ is not a rotation of (1.2). If $f(z)$ is subordinate to $F(z)$ in $D$, then $f^{\prime}(z)$ is majorized by $F^{\prime}(z)$ in $|z|<R$ for some $R>(\alpha+1)-\left(\alpha^{2}+2 \alpha\right)^{1 / 2}$, where $R$ depends only on $F(z)$ and not on $f(z)$.

\section{REFERENCES}

1. D. M. Campbell, $\beta$-close-to-linear invariant families, Dissertation, University of North Carolina, Chapel Hill, N. C., 1971.

2. —, Eventually p-valent functions, Scripta Math. 30.

3. - Locally univalent functions with locally univalent derivatives, Trans. Amer. Math. Soc. 162 (1971) 395-409. MR 44 \#4199.

4. - Majorization-subordination theory, Bull. Amer. Math. Soc. 78 (1972), 535-538.

5. G. M. Goluzin, Geometric theory of functions of a complex variable, GITTL, Moscow, 1952; English transl., Transl. Math. Monographs, vol. 26, Amer. Math. Soc., Providence, R. I., 1969. MR 15, 112; 40 \#308.

6. T. H. Gronwall, Sur la déformation dans la représentation conforme, C. R. Acad. Sci. Paris 162 (1916), 249-252.

7. W. K. Hayman, A characterization of the maximum modulus of functions regular at the origin, J. Analyse Math. 1 (1951), 135-154. MR 13, 545; 1139.

8. - Some applications of the trans-finite diameter to the theory of functions, J. Analyse Math. 1 (1951), 155-179. MR 13, 545. 
9. —- Multivalent functions, Cambridge Tracts in Math. and Math. Phys., no. 48, Cambridge Univ. Press, Cambridge, 1958. MR 21 \#7302.

10. J. Krzyz, On the maximum modulus of univalent functions, Bull. Acad. Polon. Sci. Cl III, 3 (1955), 203-206. MR 17, 143.

11. T. H. MacGregor, Majorization by univalent functions, Duke Math. J. 34 (1967), 95-102. MR 34 \#6062.

12. A. I. Markuševič, Theory of analytic functions, GITTL, Moscow, 1950; English transl., Theory of functions of a complex variable, Vol. III, Prentice-Hall, Englewood Cliffs, N. J., 1967. MR 12, 87; 35 \#6799.

13. Ch. Pommerenke, Linear-invariante familien analytischer Funktionen. I, Math. Ann. 155 (1964), 108-154. MR 29 \#6007.

14. - Linear-invariante familien analytischer Funktionen. II, Math. Ann. 156 (1964), 226-262. MR 29 \#6007.

15. - On Close-to-convex analytic functions, Trans. Amer. Math. Soc. 114 (1964), 176-186. MR 30 \#4920.

16. Tao Shah, On the radius of superiority in subordination, Sci. Record 1 (1957), 329-333. MR 20 \#6531.

Brigham Young University, Provo, Utah 84602 